NOTE

\title{
New Publication
}

The Society for Industrial and Applied Mathematics has announced the publication of a new series of its Journal to be known as Series B: Numerical Analysis.

According to the publishers, this supplementary publication will consist of research papers on the development and analysis of numerical methods dealing generally, but not exclusively, with such topics as convergence, stability, and error analysis. Also included are papers on functional analysis and closely related fields, such as approximation theory. On the other hand, descriptions and applications of numerical methods are to be retained in the existing SIAM Journal.

Volume 1, appearing in October 1964 as a single issue, consists of papers presented at the SIAM Symposium on Approximation held at Gatlinburg, Tennessee in October 1963.

Volume 2, scheduled for publication in 1965, will appear in several issues, as will subsequent volumes.

The annual subscription rate is $\$ 10.50$ ( $\$ 7.50$ to members of SIAM); orders for subscriptions should be sent to SIAM, Box 7541, Philadelphia 1, Pennsylvania. 